latindex @ideAs Econpapers oDialnet MIAR Surcupira

\title{
COMERCIO ELECTRÓNICO COMO VENTAJA COMPETITIVA DURANTE LA PANDEMIA POR COVID -19, EN LAS MIPYMES
}

\author{
Dr. Ismael Guzmán Barrera1 \\ Dra. Luz Daniela Arguelles Hernández2 \\ Lic. Jaime Antonio Rodríguez Gómezs
}

\begin{abstract}
Para citar este artículo puede utilizar el siguiente formato:
Ismael Guzmán Barrera, Luz Daniela Arguelles Hernández y Jaime Antonio Rodríguez Gómez (2021): "Comercio electrónico como ventaja competitiva durante la pandemia por COVID -19, en las MIPYMES", Revista contribuciones a la Economía (Vol 19, № 2, p.p. 76-92, febrero-julio 2021). En línea:

https://doi.org/10.51896/contrieconomia/GOFR4966
\end{abstract}

\section{RESUMEN}

El comercio electrónico, también conocido como e-commerce, término anglosajón, es un fenómeno que ha afectado, y no para mal, sino para innovar, en toda la clasificación de empresas o unidades económicas, en la forma tradicional que se tenía de hacer negocios o comercializar, desde el ámbito local hasta el internacional. Este estudio, resalta la conceptualización de la administración estratégica, bajo una breve explicación, de las cinco fuerzas de Michael Porter, su incidencia en la MiPyMe de la ciudad de Xalapa, Veracruz, México, que incluso, es de gran utilidad para fortalecer la competencia empresarial. Además de resaltar, las herramientas de las tecnologías de la información y comunicaciones, también denominadas TIC's, como son el comercio electrónico y las redes sociales, como plataformas eficientes para la innovación en logro de la comercialización digital. Pero también muestra una realidad que impactó de forma negativa la pandemia del covid-19, en el comercio local de la misma ciudad antes mencionada, que con relación a la población y la demanda de comercios que ofertan bienes y servicios. Se presenta la metodología de investigación, para la obtención de resultados, con base en la definición de los objetivos, tanto general como específicos y establecimiento de las hipótesis. Los resultados se obtienen con base en procedimientos estadísticos, de datos obtenidos por el estudio de la muestra, que refleja la realidad del e-commerce y redes sociales como parte de la innovación de la MiPyMe, en la ciudad capital del estado de Veracruz, México, como apoyo o indiferencia hacia su mercado meta.

${ }^{1}$ Licenciado en Administración de Empresas Turísticas, Maestro en Gestión de Calidad y Doctor en Educación. Docente en la Escuela de Estudiantes Extranjeros de la Universidad Veracruzana, isguzman@uv.mx

1 Licenciada en Administración de Empresas, Maestra en Gestión de Calidad y Doctora en Finanzas Públicas. Docente de licenciatura en los programas educativos de Contaduría y Administración, del Sistema de Enseñanza Abierta de la Universidad Veracruzana, darquelles@uv.mx

${ }_{1}^{1}$ Egresado de la licenciatura en Administración de Empresas, Sistema de Enseñanza Abierta por la Universidad Veracruzana, jimyrodmez@gmail.com 
Palabras clave: comercio electrónico, MiPyMe, innovación, redes sociales, contingencia.

E-COMMERCE AS A COMPETITIVE ADVANTAGE DURING THE COVID-19 PANDEMIC, IN MIPYMES ABSTRACT

Electronic commerce, also known as e-commerce, an Anglo-Saxon term, is a phenomenon that has affected, and not for the worse, but to innovate, in the entire classification of companies or economic units, in the traditional way of doing business or commercialize, from the local to the international scope. This study highlights the conceptualization of strategic management, under a brief explanation, of the five forces of Michael Porter, its incidence in the MiPyMe of the city of Xalapa, Veracruz, Mexico, which is even very useful to strengthen competition business. In addition to highlighting, the tools of information and communication technologies, also called ICTs, such as electronic commerce and social networks, as efficient platforms for innovation in achieving digital marketing. But it also shows a reality that the covid-19 pandemic had a negative impact on local businesses in the same city mentioned above, than in relation to the population and the demand for businesses that offer goods and services. The research methodology is presented, to obtain results, based on the definition of the objectives, both general and specific, and establishment of the hypotheses. The results are obtained based on statistical procedures, from data obtained by the study of the sample, which reflects the reality of e-commerce and social networks as part of the innovation of the MiPyMe, in the capital city of the state of Veracruz, Mexico, as support or indifference towards your target market.

Keywords: e-commerce, MiPyMe, innovation, sociales network, contingency

\section{INTRODUCCIÓN}

Las empresas o unidades económicas son la fuerza motora de producción del comercio al interior del país, la innovación de ellas, seguirá determinando la preferencia del público y permanencia en el mercado. Así que las Micro, Pequeñas y Medianas empresas (MiPyMe), que deseen tener una presencia en internet, deberán romper barreras, y entender la tecnología, presentándose al mercado con una nueva herramienta, que es el comercio electrónico, también conocido como "e-commerce".

Analizar a la ciudad de Xalapa, Veracruz, bajo el contexto económico, hace reflexionar sobre todas las posibilidades de exploración comercial, y claro está, con beneficios al interior; ya que la Atenas Veracruzana, hoy por hoy, tiene un crecimiento de población exponencial, pues para este año, 2020, se alcanzaba aproximadamente la cantidad de medio millón de habitantes, entre población nativa y flotante. $Y$ sin duda, este crecimiento demanda todo tipo de actividades comerciales, pero que lamentablemente este mismo año se frena por la contingencia sanitaria, y la incertidumbre de todo empresario, es como enfrentar el déficit comercial por ventas bajas o nulas. 
El objetivo general de esta investigación es analizar la innovación dentro de las MiPyMe al haber tomado la iniciativa de crear un comercio electrónico o plataforma afín, antes o durante, los meses de marzo a junio de 2020, cuyo análisis de enfoque cualitativo, que se logrará a través de la aplicación de la herramienta denominada cuestionario, cuya muestra de unidades de análisis se estudiarán en la ciudad de Xalapa, Veracruz.

Se establecen dos hipótesis en este estudio y que se resuelven a través de la recolección de datos, un análisis en profundidad y de la aplicación de procesos estadísticos. En donde la primera hipótesis, de tipo de investigación causal, enuncia que: La innovación empresarial es un concepto claro, que tienen los encuestados de las unidades económicas de la muestra tomada de la ciudad de Xalapa, Veracruz; que desarrollan al trabajar en esta situación emergente de la actual contingencia sanitaria.

En la segunda hipótesis, de investigación descriptiva de un dato, o de valor que se pronostica, se declara que: El comercio electrónico ha logrado innovar a las MiPyMe de Xalapa, Veracruz, en relación a su mercado, durante los meses de marzo a junio de 2020.

Este trabajo utiliza sugerencias, del libro de Metodología de la investigación (Hernández, 2010), sobre el enfoque cualitativo, utilizando una muestra, integrada por once unidades de análisis, conformada por MiPyMe, de la ciudad de Xalapa, Veracruz; siendo estas de cualquier giro o actividad comercial, según lo permita la regulación de la nueva normalidad.

Durante el recorrido del marco teórico, se conocerá la conceptualización de innovación empresarial, se analizará a la vez, la teoría de Michel Porter, en materia de estrategias para la ventaja competitiva empresarial, se analizarán dos herramientas de las TICs, comercio electrónico y redes sociales, como parte de la innovación tecnológica para las empresas y estrategia de atención a su mercado. Se enunciarán algunas ventajas del Tratado de México, Estados Unidos y Canadá (TLCAN), firmado en 1994 y el Tratado entre México, Estados Unidos y Canadá (T-MEC), el cual entró en vigor el 1 de julio de 2020, en materia de comercio internacional, así como las modalidades del comercio electrónico y la clasificación de las MiPyMe.

El marco referencial, estudia el contexto actual que se vive en la ciudad de Xalapa, Veracruz, al momento de realizar la investigación. Se inicia con un enfoque del e-commerce en México, y las acciones de la Asociación de internet México, "AIMX", quien provee información sobre distintas temáticas en el contexto digital, en la república mexicana, de esta misma, se expone el estudio de las tendencias de compra virtual, por la ya mencionada, que existen entre los internautas en el año 2019; Y, por último, una entrevista con Studio Creativo Xalapa, agencia de e-marketing o marketing digital, que nos hace reparar en la realidad del comercio electrónico en las MiPyMe de esta misma ciudad. 
Para el marco metodológico, se resolverán la cuestionantes que exigen, tanto los objetivos e hipótesis, establecidas en este estudio. Lo que resolverá en forma definitiva si las empresas xalapeñas innovan durante este tiempo, además, de saber si se benefician de las nuevas tendencias tecnológicas que marca la digitalización. Los resultados podrán ser captados, a través de los análisis de datos obtenidos de la muestra por once unidades de análisis estudiadas en el campo, de esta ciudad de Xalapa, Veracruz; y se reforzará aún más su interpretación, cuando se estudien a la luz de la aplicación de técnicas estadísticas y gráficas. Las conclusiones de este estudio nos darán la pauta para entender, que estrategias se necesitan implementar en la práctica comercial de las unidades económicas para eficientar su competencia en el mercado.

\section{DESARROLLO}

\section{EL E-COMMERCE EN LA INNOVACIÓN DE LAS MICRO, PEQUEÑAS Y MEDIANAS EMPRESAS}

En este siglo XXI, sin duda, vivimos en un entorno competitivo y al mismo tiempo globalizado, en donde las MiPyMe que, con seguridad, avanzan, son aquellas que están resueltas a innovar, es decir, aquellas que están atentas a los cambios del entorno y responden a ellos, con objetivos muy claros. Por lo tanto, existen muchas formas de innovar, y una de ellas es a través de la tecnología. ¿Qué es innovación empresarial? Westreicher (2017) expresó sobre este tema, "la innovación empresarial es un cambio que se introduce al bien o servicio que ofrece una compañía, o a los procesos que se desarrollan dentro de la organización. Esto, a fin de alcanzar mayor competitividad".

El Dr. Xavier Amores Bravo, de la Universidad de Girona, España, en su tesis, aborda el tema de la innovación, señalando, del año 1998, a Cristopher Freeman, quien resalta algunas carencias en el tratamiento económico de la innovación, entre otros puntos, analiza el aprendizaje tecnológico a partir de fuentes externas, es decir, diferentes mecanismos, en donde las empresas adquieren conocimientos científicos y tecnológicos que facilitarán el proceso de innovación (Amores, 2015). La importancia, entonces, que reviste este primer subtítulo de esta aportación es porque parte del interés es analizar a la MiPyMe xalapeña, en el contexto de la innovación empresarial, con el fin de indagar el concepto que tienen de la misma, así como saber, cuál es su comportamiento bajo este mismo contexto, incluso, en eventos emergentes como la crisis económica que vive el comercio nacional y mundial en este año 2020.

\section{Las cinco fuerzas Michael Porter: ventaja competitiva}

Joan Magretta, quien es autora de varios libros sobre la temática de estrategias y modelos de negocios; y egresada de Harvard en los años ochenta, trabajó con clientes de varios giros, así como en empresas no lucrativas y siempre analizaba el progreso o declinamiento de las mismas. Esto anterior, la motivó a seguir la metodología de Michael Porter, la inspiró a escribir el libro, "Para comprender a Michael Porter - 
guía esencial hacia la estrategia y la competencia". Esta metodología, herramienta para la planeación estratégica empresarial, tiene el objetivo de impulsar a las unidades económicas en su administración estratégica (Magreta, 2014).

La anterior metodología, no es un tema aislado, pues algunos actores que han hecho aportaciones al Instituto de Investigaciones y Estudios Superiores de las Ciencias Administrativas de la Universidad Veracruzana, "IIESCA UV", como son el Académico de la Facultad de Contaduría y Administración de Universidad Veracruzana, Jorge Iván Ramírez Sandoval y al Consultor de negocios Enrique Federico Gochicoa Gramer, quienes han señalado a Michael Porter, en su estudio presentado, "Imagen corporativa: ventaja competitiva para las organizaciones PYME", cuando exponen de las cadenas de valor y ventaja competitiva (Ramírez, 2010)

Se expone a continuación las cinco fuerzas del autor ya mencionado, que se extraen del libro "Para entender a Michael Porter - Guía esencial hacia la estrategia y la competencia” (Magreta, 2014): el nivel de intensidad de la competencia, la competencia potencial o amenaza de nuevos aspirantes, el poder de negociación de los clientes, el poder de negociación de los proveedores, cantidad de productos sustitutos.

\section{Los medios de comunicación y clasificación}

Los medios de comunicación son los instrumentos a través de los cuales se informa y se comunica de forma masiva. Los medios de comunicación son el canal mediante el cual la información se obtiene, se procesa y, finalmente, se expresa, o se comunica. Pazmiño (2010), expone en su tesis de comunicación social, grado licenciatura, una clasificación tanto en estructura física como de estructura de carácter de la información.

La clasificación de los medios de comunicación por estructura física serían los siguientes: medios audiovisuales, medios radiofónicos, medios impresos, medios digitales.

La clasificación de los medios de comunicación por estructura carácter de estas serían las siguientes: estructura informativa, estructura de entretenimiento, estructura de análisis, estructura especializada, estructura comercial

Los medios de comunicación utilizados para comercio en Xalapa, Veracruz, son legales, como en cualquier parte de nuestro país, puede ser de cualquier estructura física ya que son ampliamente utilizados en estos campos, y de carácter comercial y bajo este contexto hablamos de estructura física digital y carácter comercial. 


\section{Redes sociales en MiPyMe medio de comunicación actual}

Las redes sociales en Internet han ganado terreno de una manera exponencial aportando valor de imagen, pero más importante aún, el valor monetario para empresas y sobre todo para relaciones sociales y encuentros para humanos. La Lic. Oti Elizabeth Mejía Cruz, en su tesis de comunicación social, comparte la siguiente definición de redes sociales: "Se llama a la evolución de las tradicionales maneras de comunicación del ser humano, que han avanzado con el uso de nuevos canales y herramientas" (Mejía, 2012), existen diversas aplicaciones que aluden a las redes sociales, por poner ejemplo, facebook, twiter, instagram, whatsapp, y tik tok más recientemente, entre otras. Si bien las redes sociales siempre han existido desde los inicios de la humanidad, y han evolucionado, con base a nuevas necesidades; con la aparición del teléfono inteligente, Smartphone, y el internet, se eficientaron las redes sociales para uso comercial teniendo gran desempeño lucrativo y legal.

¿Redes Sociales efectivas para MiPyMe de Xalapa, Veracruz?

Mejía (2012) abordó el tema de la posibilidad de comercializar los bienes y servicios por Facebook y el resultado estadístico compartido fue, que el $85 \%$, dijo que era correcto utilizar Facebook para fines comerciales. Marcando con esto, un antecedente para las MiPyMe xalapeñas, en lo relacionado a trabajar con redes sociales, en términos de publicidad para comercializar un bien o servicio.

\section{Comercio Internacional: T-MEC}

¿Qué se entiende por comercio? Tello (2014) afirma que "El comercio, se entiende como toda actividad que busca generar un intercambio de bienes y servicios valuables entre dos agentes, y todas aquellas transacciones realizadas para poder llevarlo a cabo, esta relación llevada al término económico, nos refiere a los procesos de compra-venta". Se deduce que es toda actividad que busca generar un intercambio de bienes y servicios de valor entre dos agentes, llámese entre personas, instituciones privadas y gobierno; y que tienen como objetivo fortalecer la economía de un país.

Ahora bien, si hablamos de comercio internacional, partamos de lo actual, el T-MEC, que sigue siendo México, Estados Unidos y Canadá, firmado en Argentina en noviembre de 2018, y ratificado por los Estados Unidos de América el diez de diciembre de 2019, entrando en vigor el primero de julio de 2020. Pero ¿Qué logra el T-MEC, en materia de comercio internacional? Mantiene la apertura en el comercio de bienes y servicios e incorpora nuevas disposiciones en materia de reglas de origen, comercio electrónico, empresas propiedad del Estado, derechos laborales y ambientales, corrupción, mecanismos de solución de controversias y propiedad intelectual, además de, implementar cambios notables en las disposiciones de acceso al mercado para autos y productos agrícolas, y en reglas tales como la inversión, la contratación pública y los derechos de propiedad intelectual (Opportimes, 2019). Por otro lado, México, está abierto a la apertura comercial, no tan solo por el T-MEC, ya que actualmente tiene acuerdos comerciales o tratados de libre comercio con cincuenta países. 
Contexto actual del comercio en Xalapa, Veracruz

La situación actual del comercio en la ciudad de Xalapa, Veracruz, se presenta como lo expresa un periódico de gran circulación en el estado de Veracruz, cuyo contenido se hace responsable por la veracidad de sus líneas, con fecha del jueves cuatro de junio de 2020, en donde el presidente de la CANACO-SERVYTUR, Bernardo Martínez Ríos, indica que posiblemente un 30\% de los comercios que cerraron de forma preventiva a causa de la pandemia por coronavirus no podrían reactivarse, o en su defecto, lo harían de forma muy limitada, una vez que pasara la contingencia (Xalapa, 2020).

Se puede deducir, por la situación actual que muchas empresas de la ciudad de Xalapa, Veracruz, tendrán serias desavenencias, en lo económico, sin duda, para estas unidades económicas, serán las de mantener, aún a puerta cerrada, su plantilla de fuerza laboral, con un sueldo o salario, el pago de rentas de los establecimientos ocupados, créditos obtenidos por medio de financieras o instituciones bancarias y pago de seguridad social para sus empleados. (Xalapa, 2020).

\section{Situación de internet nacional y local}

Los proveedores destacados, de internet, en la república mexicana son: Axtel, Megacable, Izzi, Infinitum (de Telmex), Total Play. Pero ¿Qué demanda se dio entre los años 2019 y 2020, en el servicio de internet a nivel nacional? según reporte del Instituto Nacional de Estadística y Geografía e Informática (INEGI) en colaboración de la Secretaría de Comunicaciones y Transporte (SCT) y el Instituto Federal de Telecomunicaciones (IFT), en México, existe 80.6 millones de usuarios de este servicio, lo que representa un $70.1 \%$ de la población de seis años o más. Así mismo, este reporte informa que los tres principales medios para la conexión de usuarios a Internet en el año 2019 fueron: celular inteligente Smartphone con $95.3 \%$, computadora portátil con $33.2 \%$, y computadora de escritorio con $28.9 \%$.

Ahora bien, ¿qué se puede decir para Xalapa, Veracruz? y ¿cuán efectivo resulta ser del servicio de internet? Ésta entidad federativa, sufre un rezago en el acceso a internet, como un medio de información o consulta, incluso para temas laborales o académicos. Así mismo, el periódico Diario de Xalapa informa en su sección de finanzas, del miércoles tres de abril de 2019: "Veracruz se ubicó entre las cinco entidades del país con el menor número de usuarios de Internet, tanto en zonas rurales como en urbanas, reveló la ENDUTIH del INEGI" (Xalapa, 2019).

\section{Comercio electrónico ¿Qué es y cómo afecta nuestra vida?}

El comercio electrónico se puede entender como "el uso de la transmisión electrónica de datos para llevar a cabo y/o incrementar los procesos comerciales". Este tipo de comercialización va de la mano con la globalización, generando con ello una gran atracción a nuevos mercados, y las empresas están incurriendo en la puesta en marcha de él como parte de sus estrategias (Tello, 2014). 
La manera que nos afecta, que definitivamente positiva, es cuando se gana terreno sobre la forma tradicional de hacer negocios, afectando la distribución, pues las tiendas en línea estarán disponibles, las 24 horas del día, en los 365 días del año, creando con ello una reducción en las cadenas de intermediarismo. Así mismo, nos referimos al municipio, de Xalapa, Veracruz, que no es la excepción, ya que tiendas grandes de nuestra comunidad, crean e-commerce y se dedican a la distribución local, por este motivo, la investigación que se desarrollará en esta tesis, nos indicará si realmente las MiPyMe que existen en Xalapa, Veracruz, en la actualidad, tienen un verdadero compromiso con dar a conocer su marca y bienes al mercado local, a través del comercio electrónico.

México y Veracruz en el comercio electrónico

Parece paradójico, un rezago que tiene el estado de Veracruz al acceso de internet bajo el contexto de buscar información a manera de consulta o investigación, a que México como país, este teniendo repuntes año con año en el incremento de internautas, pero específicamente las cifras de consulta o compras del comercio electrónico tuvieron un aumento constante en los últimos años y se espera que la evolución continúe así. Pues mucho se debe al comportamiento del público consumidor, que está cada vez más conectado.

Según un informe de Digital 2019 México, creado por hootsuite, que es una aplicación web y móvil que sirve para gestionar redes sociales para personas u organizaciones; quien trabaja con Google Analytics y Facebook Insights, indica que para el año 2019, del total de población mexicana de 131,500,000 de personas, 88,000,000 representó la población urbanizada o 67\% y de estos, los que usaron e-commerce solo fue el $13 \%$ de la misma población (hootsuite, 2019).

Además, según este mismo informe: Digital 2019 México, los segmentos que más han crecido en ventas a través de Internet son (hootsuite, 2019): Viajes 21\%, Muebles y Electrodomésticos 19\%, Juguetes y Hobbies 19\%, Moda y belleza 16\%, Electrónicos y Medios Físicos 12\%, Alimentos y Cuidado Personal $11 \%$, Música Digital 8,9\%, Videojuegos 8,6\%. Lo anterior supone un gran campo de acción, ya que los porcentajes nos dicen acerca de los segmentos en que las MiPyMe xalapeñas pudieran estar interesadas en trabajar con un comercio electrónico.

Por otro lado, la CANACO-SERVYTUR en Xalapa, Veracruz, quien en apoyo a todas las actividades comerciales e impulso de las MiPyMe, así como asesoramiento a través capacitaciones técnicas, en varios aspectos de la vida de las unidades económicas, anunció para el siete de mayo de 2020, la creación de una aplicación móvil comprafácil con la finalidad de incentivar el comercio entre los micro, pequeños y medianos empresarios (Camarillo, 2020), así mismo, esta información fue publicitada por otras fuentes. 


\section{LAS “MIPYME” DE XALAPA, VERACRUZ Y SU REALIDAD EN EL COMERCIO ELECTRÓNICO}

\section{La economía nacional afectada por la pandemia este año 2020}

Hay una realidad innegable, que se vive actualmente en todo el mundo, por consiguiente, México y, por ende, sus entidades federativas, con afectación económica por la pandemia sanitaria covid-19. Una plataforma digital de noticias, promovida incluso por los medios radiofónicos, el demócrata, publicó en su sitio web, el once de agosto de 2020, lo siguiente: "el ex diputado local Carlos Morales Guevara señaló que hasta el momento se tienen cifras de que al menos 93.2 por ciento de los pequeños negocios en el país están en proceso de bajar sus cortinas." Este ex diputado local de la ciudad de Xalapa, Veracruz, hizo mención de otro punto importante al respecto, que más adelante se dará a conocer (Paredes, 2020). Derivado de lo anterior, pues más de quince millones de personas, han perdido su empleo, además de que otros, es decir, nueve millones trabajan con la mitad de su salario, ambos datos a nivel nacional.

\section{La realidad del e-commerce en México}

Con base en una tesis de pregrado maestría de la UNAM, en su página 12, se indica que, en nuestro país, el e-commerce, desde su aparición hasta por lo menos para el año 2011, los consumidores habían aceptado a este mismo de una forma lenta (Portilla, 2011).

Los motivos se exponen a continuación: Primero, un comercio electrónico no otorgaba confianza para compartir datos personales y la segunda, el temor de compartir información de la tarjeta de crédito. Las siete causas seguidas son: la incertidumbre de que el producto no llegara, no contar con una tarjeta de crédito, la falta de interés al comprar en un comercio electrónico, elevados gastos de envío, no entender el proceso de compra de un comercio electrónico, falta de opciones en el e-commerce, los tiempos muy largos para el arribo al domicilio solicitado.

La tesis antes mencionada, da como referencia a la AMIPCI, Pero ¿Qué es la AMIPCI? La información que otorga el investigador mencionado es del año 2011, ésta cambió de denominación social, para la fecha del primero de diciembre de 2016, al nombre de Asociación de internet.mx, esto anterior, debido a sumarse a su lista más empresas y actores (Velazquez, 2016).

\section{La Asociación de Internet.Mx: AIMX}

La AIMX, es una asociación civil mexicana que trabaja con los principales actores de la industria de internet. Maneja información de una amplia temática del mundo digital, es un marco de referencia para el desarrollo e implementación de proyectos normativos y de política pública para contribuir en la productividad y la competitividad de México (AIMX, 2019). Portilla (2011), expone en su tesis, las funciones que desempeña, la hoy llamada AIMX. 
- Fomentar el uso, responsable y productivo, de los medios digitales por parte de empresas, instituciones y usuarios.

- Realiza estudios y eventos anuales permitiendo conocer las tendencias de actualidad y la percepción de los usuarios bajo el contexto del Internet.

- Colabora directamente con organizaciones aliadas, autoridades, funcionarios, legisladores, miembros de la academia, la industria y de la sociedad civil por un debido aprovechamiento y uso responsable de Internet, así como de las TICs.

Un mercado mexicano cambiante a una década

¿Actualmente cuánto ha cambiado el mercado mexicano, respecto a la experiencia de comprar en un ecommerce? Esto anterior, debido a que la información que expuso el Maestro Omar Portilla Sánchez, fue de diez años atrás, ahora bien, en este año 2020 ¿cuál es el comportamiento del consumidor mexicano respecto a esta actividad comercial digital? De la misma fuente se expresa lo siguiente, si para 2010, el volumen de ventas había sido de 36,500 millones de pesos, para 2019, y por lo menos, lo que refleja la misma AIMX, para los primeros seis meses, fue de 299,660 millones de pesos, lo que denota un crecimiento exponencial (MX, 2019). De lo anterior, ¿Cuál es la disposición del mercado mexicano, para seguir evolucionando ante una respuesta de cambios tecnológicos e incluso digitales? ¿Será que en todas y cada una de las entidades federativas que conforma la república mexicana, tengan la misma conducta de valoración al e-commerce?

\section{Tendencias o comportamientos de compra en e-commerce}

Los datos otorgados por la AIMX (2019), son interesantes y muy retroalimentativos, permite analizar los comportamientos de compra y pago de los internautas, en sus hábitos de consumo, para que cualquier empresario mexicano, esté alerta y vea en ellos las posibilidades de innovación en sus negocios. Llama la atención que, en el tema de liquidez, el 63\% de los consumidores están dispuestos a pagar de una sola vez sin la utilización de un apoyo bancario, lo que reporta inmediatez en flujo monetario para empresarios locales o xalapeños que requieran capitalizarse de forma inmediata. Es interesante estos datos, por ejemplo, el primer punto, señala que de un 100\% que buscaron por internet algo de su interés, el 85\% terminó comprando por medio de su Smartphone, a través de una app; esto es una herramienta básica para todo aquel que quiera incursionar en este modelo de negocios por internet, el crear una app, que pueda ser visitada por parte del internauta para cerrar en ese momento una compra. Algo que al final de este marco se analizará.

Por último, la predisposición de compra o repetir la misma experiencia de compra por internet, al indicar que el $84 \%$ tiene una satisfacción de compra por este medio y que el $91 \%$ volvería a comprar en el año 2020, permitiendo a un empresario xalapeño, el grado de éxito con un e-commerce.

\section{Respuesta del mercado Veracruzano al e-commerce}


De acuerdo con los datos del INEGI (2019), las MiPyMe tuvieron en cinco años, del año 2014 al año 2019, un crecimiento en la entidad, reportando la cantidad de 276 mil 739 unidades económicas, en la clasificación de MiPyMe. De lo anterior como base, la Secretaria de Desarrollo Económico y Portuario (2020), "SEDECOP", del Estado de Veracruz, dio a conocer en marzo del presente año, a través de su titular, Enrique Nachón García, que el estado prepara la primera edición de "Hecho en Veracruz".

¿En qué consiste la edición? De acuerdo a lo que comenta la agencia de noticias Radio, Televisión de Veracruz, "RTV", en su página virtual, es que es un catálogo virtual, con actualización diaria, mejor denominado comercio electrónico. el paso inmediato sería el posicionamiento de su producto en tiendas locales para la venta del producto y en la parte internacional sería a través de las embajadas. Esto anterior, nos debe hacer pensar en el hecho de que las empresas veracruzanas pudieran aprovechar la oportunidad de expandirse a través de este medio electrónico innovador (RTV, 2020).

\section{Conducta del mercado xalapeño frente a e-commerce}

¿Cómo enfrenta la ciudad de Xalapa, Veracruz, la economía en este periodo de tiempo, en que los negocios se ven afectados por la baja afluencia de gente en las calles, o incluso de los que eran usuarios normalmente a un tipo de giro? el día trece de agosto de 2020, se entrevistó al Lic. Santos Serra, quien en ese momento se desempeñaba como gerente de sucursal en Xalapa, Veracruz, y cuya matriz se encuentra en la ciudad de Monterrey, de la agencia, Studio Creativo Xalapa, con la prestación de servicios de estrategias en e-marketing y publicidad, en la elaboración de diseño web y redes sociales, y con experiencia de cuatro años en esta ciudad.

La realidad que comenta, Santos Serra, para la ciudad de Xalapa, Veracruz en el contexto de comercio electrónico, diciendo que es un área de oportunidad muy importante. Puede parecer una desavenencia lo siguiente, pero nos hace reparar en un análisis de costo de oportunidad, cuando dice, "este asunto de comercio electrónico se convirtió en un tema de recaudación de impuestos a partir del uno de julio de este año", pero Santos Serra comenta lo siguiente, "sin embargo, plataformas como mercado libre, spotify y amazon, aparte de la comisión que se le tenga que pagar, como un aproximado de 5\%" por venta, hay que realizar un pago de impuestos que cobre la misma plataforma por temas fiscales, por lo que "la utilidad que le queda al vendedor es muy corta". En lugar de ello, si el negocio tiene su propia página web y lo combinara con la publicidad en redes, el margen al final sería mayor.

Se le preguntó ¿Qué es lo que ha hecho la agencia Studio Creativo Xalapa al respecto?, y compartió que es el crear campañas por Facebook, para promocionar los beneficios que se derivan de tener una página web y de analizar con los pequeños comerciantes, las desventajas de trabajar en plataformas de 
multinegocios, como las ya mencionadas ¿Qué pueden hacer las empresas pequeñas para que puedan trabajar eficientemente?

En conexión con sus mayoristas, por medio de una estrategia, bajo el sistema de drop shiping, en donde sólo el comerciante es el contacto del usuario final, por medio de su página y el mayorista envía el producto a este destino, evitando tener mercancía innecesaria y en el tema de las redes sociales, la agencia de Studio Creativo Xalapa, analiza que en el mercado xalapeño, resulta favorable comercializar en grupos de venta por facebook o incluso por el comercio de la dirección de desarrollo económico.

Serra (2020) destaca que para Xalapa, Veracruz, es más directa la comercialización, que, si bien utiliza las redes sociales, le resulta más efectivo ofrecerlo directamente al cliente mostrando fotos y ofreciendo el servicio, es decir, existe carencia de cultura digital. No obstante, las empresas pequeñas bien asesoradas y sobre todo lo que está haciendo la dirección de desarrollo económico, con este tipo de capacitaciones para las micro y pequeñas empresas, es como un área de oportunidad muy grande.

\section{METODOLOGÍA PARA CONOCER LA INNOVACIÓN EN LA MIPYME}

\section{Planteamiento del problema}

CNN Español, da a conocer el catorce de abril de 2020, que el Fondo Monetario Internacional, "FMI", "confirma que debido al covid-19 este 2020, viviremos la peor recesión económica en 90 años, superando lo visto durante la crisis financiera global de 2008. En donde México, tendrá una contracción en su economía del 6.6\%. El FMI indica que, las nuevas tecnologías digitales pueden ser útiles para localizar los apoyos (Frías, 2020). Por lo anterior se plantearon las siguientes preguntas: Las unidades de análisis encuestadas que conforman la muestra en la ciudad de Xalapa, Veracruz; ¿entienden el concepto de innovación empresarial, cuando se vive y trabaja, bajo situaciones emergentes, como la actual contingencia sanitaria? ¿Es el comercio electrónico lo que logra innovar a las MiPyME de Xalapa, Veracruz; en su mercado principalmente en los meses de marzo a junio de 2020 ? ¿las MiPyME de Xalapa, Veracruz, han creado nuevas formas de contacto y servicio con su mercado, en lo relacionado a la comercialización digital?

\section{Objetivo general}


Analizar la innovación dentro de las MiPyMe al haber tomado la iniciativa de crear un comercio electrónico o plataforma afín, antes o durante, los meses de marzo a junio de 2020, cuyo análisis se logrará a través de la aplicación de la herramienta propuesta, según lo permitan las circunstancias bajo la norma de contingencia sanitaria y no proximidad, cuya muestra de empresas se estudiará en la ciudad de Xalapa, Veracruz.

\section{Delimitación de la investigación}

El interés de estudio son once MiPyMe de actividad comercial indistinta para estudio, ubicadas dentro de la ciudad de Xalapa, Veracruz y se analizó la exposición del comercio electrónico, así como explicación de las redes sociales, y un análisis actual de la economía, durante el periodo de marzo a junio de 2020.

\section{Población y muestra}

Para esta investigación, se consideró como población o universo al total de unidades económicas o negocios registradas en la ciudad de Xalapa, Veracruz, siendo estas la cantidad de 26,136 unidades económicas. En relación al tamaño de muestra y debido a que es una investigación de enfoque cualitativo, se basa el criterio a lo referido por Hernández (2010), "después de la inmersión inicial: la muestra inicial", nos dice que el tamaño de muestra en un estudio cualitativo no es importante desde una perspectiva probabilística, pues no se van a generalizar los resultados de su estudio a una población más amplia, lo que nos debe interesar es a entender el fenómeno de estudio y a responder a las preguntas de investigación.

\section{Instrumentos de recopilación}

Para la recolección de datos y estudio de los casos de las unidades de análisis que conforman la muestra, se utilizó la técnica de encuesta a través de la herramienta de cuestionario (Tamayo, 2015). Además, que en los casos donde no hubo participación para aplicación de cuestionario, pero si una plática informal, se tomaron datos de observación, y se vertieron en un formato de bitácora de campo, utilizándola como otra herramienta de recolección.

\section{RESULTADOS}

Derivado del estudio realizado se concluyó que no todas las unidades de análisis de la muestra concilian el mismo concepto, e incluso de forma clara, pues algunos de ellos, guardan ignorancia, en el sentido de desconocer de qué forma es aplicativa la innovación en la empresa. Solo el $64 \%$ de los encuestados pudo dar una respuesta lógica y acertada y $36 \%$ supo indicar las pautas que siguieron para trabajar bajo ese contexto.

Respecto a la innovación de comercio electrónico en las MiPyMe, Para estas unidades de análisis estudiadas, el e-commerce no es un tema nuevo, pues la interpretación estadística de la mediana, valor 
central, indique que el $50 \%$ de los casos analizados tengan una experiencia en e-commerce, de por lo menos cinco años. En donde, siete de los once casos, ya contaban con un comercio digitalizado.

Nueve de once unidades encuestadas, hacen uso activo de las redes sociales (facebook, whatsapp, twiter, Instagram, youtube y tik tok) como nueva forma de contacto con el mercado; y una determinación de valores centrales, que es la mediana, en su interpretación indica que: el 50\% de los casos estudiados, que se encuentran actualizados en TICs, es igual o menor a cuatro áreas de uso, en donde se incluyen a las aplicaciones o redes sociales.

Se determinó que ocho de once casos estudiados, podían dar ideas respecto a innovar; pero también, por otro lado, un $64 \%$, sabía lo que es innovar y en qué aspectos, mientras que un $36 \%$, sabía lo que era innovar bajo un periodo de contingencia o de crisis económica.

Si bien es cierto, que mucho antes de marzo de 2020, las unidades de análisis consideradas, ya contaban con un e-commerce y redes sociales, en donde, seis de once casos, ya contaban por lo menos desde hace cinco años con redes sociales, y un $37 \%$ de los casos le dio prioridad al comercio electrónico y $18 \%$, a las redes sociales, como parte de un tema de suma importancia en el desarrollo empresarial.

\section{CONCLUSIONES}

El principal propósito de este este documento fue analizar la innovación dentro de las MiPyMe al haber tomado la iniciativa de crear un comercio electrónico o plataforma afín, antes o durante, los meses de marzo a junio de 2020, cuya muestra de empresas estudiandas fueron en la ciudad de Xalapa, Veracruz, para ello se utilizó la técnica de encuesta a través de la herramienta de cuestionario. Además, que en los casos donde no hubo participación para aplicación de cuestionario, pero si una plática informal, se tomaron datos de observación, y se vertieron en un formato de bitácora de campo, utilizándola como otra herramienta de recolección.

Derivado del análisis realizado se concluye que para las unidades de análisis, el concepto de innovación empresarial ha implicado un enfoque de modernización al interior de ellas y en lo que ofertan. Para seguir en contacto con su mercado, han hecho uso de la digitalización: como el e-commerce y las redes sociales. La oportunidad de modernización es algo que está a la mano de cada unidad de análisis. Este estudio implicó una sensibilización de los entrevistados, que en realidad tenían poco tiempo para dar información. Se realizó observación en espacios en donde realizaban sus actividades simultáneamente a su tiempo prestado. Por lo que este estudio, permitió conocer el concepto de innovación empresarial bajo eventualidades emergentes en las unidades tratadas. 
Durante los meses de marzo a junio de 2020, 8 de 11 Mipymes de Xalapa Veracruz, ya contaban con un comercio electrónico en funcionamiento, y manejo de redes sociales para publicidad, esto anterior, como herramientas de innovación, lo que les permitió soportar el cierre temporal por contingencia.

Y por último, las Mipymes estudiadas crearon desde ya desde hace más de siete u ocho años aproximadamente, nuevas formas de contacto y servicio con su mercado, en lo relacionado a la comercialización digital, es decir, a través de las redes sociales nueve de los once casos hacen uso de una hasta seis redes sociales. El uso de redes sociales, no se encuentra limitado a nadie, cual más MiPyMe que desee hacerlo, no importando el giro, solo requiere de creatividad para la publicidad.

La herramientas utilizadas en esta investigación, permitió que los participantes externaran el hecho de saber en qué aplicaciones se publicitaban o incluso bastaba con ver alguna publicidad del caso de la muestra, para saber en qué redes se les podía consultar, no se encontró mayor dificultad. Estas redes se han convertido en una forma más de comunicación, porque estas son visitadas diariamente.

\section{BIBLIOGRAFÍA}

Amores, X. (2015). La gestión de la innovación en las empresas de servicios intensivas en conocimiento tecnológico (T-KIBS) de Cataluña [Tesis de pregrado doctoral. Universitát Girona, Cataluña]. http://hdl.handle.net/10803/380897

Camarillo, Á. (2020). Xalapa alista su app de ventas en línea. CANACO https://www.alcalorpolitico.com/informacion/canaco-xalapa-alista-su-app-de-ventas-en-linea315910.htm|\#.XvpLdChKjlU

Chunga, M. (2010). Planificación estratégica para una empresa de servicios contables. [Tesis pregrado Maestría. Universidad de Chile. Santiago de Chile]. http://repositorio.uchile.cl/handle/2250/102207

Instituto Nacional de Estadística y Geografía INEGI (2020). En méxico hay 80.6 millones de usuarios de internet y 86.5 millones de usuarios de teléfonos celulares: endutih 2019. Comunicado de prensa núm. 103/20. Recuperado de https://www.inegi.org.mx/contenidos/saladeprensa/boletines/2020/OtrTemEcon/ENDUTIH 2019. $\underline{\mathrm{pdf}}$

Frías, G. (2020). Recesión económica en 90 años. https://cnnespanol.cnn.com/2020/04/14/el-fmiconfirma-que-en-2020-viviremos-la-peor-recesion-economica-en-90-anos/ 
Hernández, R. (2010). Metodología de la investigación. 5a. Edición. México, D.F. McGrawHill/Interamericana Editores SA de CV.

Hootsuite. (2019). Digital 2019: México. https://datareportal.com/reports/digital-2019-mexico

Magreta, J. (2014). Para entender a Michael Porter - Guía esencial hacia la estrategia y la competitividad. México, DF. Grupo Editorial Patria.

Mejía, O. (2012). Uso de las redes sociales como medio comercial. [Tesis de pregrado Licenciatura). Universidad de San Carlos de Guatemala. Nueva Guatemala de la Asunción]. http://biblioteca.usac.edu.gt/tesis/16/16 1059.pdf

Asociación de Internet MX (2019). Estudio sobre Comercio Electrónico en México 2019 Décima tercera entrega. Recuperado de https://irpcdn.multiscreensite.com/81280eda/files/uploaded/Estudio\%20de\%20Comercio\%20Electro\%CC\% 81nico\%20en\%20Me\%CC\%81xico\%202019.pdf

Opportimes, R. (19 de diciembre de 2019). T-MEC señal al mundo de apertura comercial de México. https://www.opportimes.com/da-t-mec-senal-al-mundo-de-apertura-comercial-de-mexico/

Paredes, Y. (11 de agosto de 2020). Necesario promover el consumo local para reactivar la economía. https://eldemocrata.com/necesario-promover-el-consumo-local-para-reactivar-la-economia/

Pazmiño, P. (2010). El impacto de las redes sociales y el internet en la formación de los jóvenes de la Universidad Politécnica Salesiana: Caso carrera de comunicación Social Sede Quito. [Tesis pregrado Licenciatura Universidad Politécnica Salesiana. Quito]. http://dspace.ups.edu.ec/handle/123456789/2618

Portilla, O. (2011). Factores Críticos de Éxito en el Comercio Electrónico; El caso de los Negocios Tipo B2C. [Tesis de pregrado maestría. UNAM. México, D.F.] http://www.ptolomeo.unam.mx:8080/xmlui/handle/132.248.52.100/4735

Ramírez, J.; Gochicoa, E. (2010). Imagen corporativa: ventaja competitiva para las organizaciones PYME. https://www.uv.mx/iiesca/files/2012/12/imagen2010-1.pdf 
Radio Televisión de Verecaruz RTV (2020). Mediante comercio electrónico Sedecop posicionará la marca "Hecho en Veracruz". http://www.masnoticias.mx/mediante-comercio-electronico-sedecopposicionara-la-marca-hecho-en-veracruz/

Secretaria de Economía. (2019). Industria y comercio. https://www.gob.mx/se/acciones-yprogramas/industria-y-comercio

Serra, S. (2020). Conducta del mercado xalapeño frente a e-commerce. (J. A. Rodríguez, Entrevistador)

Tello, J. (2014). Estudio de factores que permean el uso del comercio electrónico como herramienta de comercialización en las PyME y la cultura de compras por internet en la población de Xalapa, Ver. [Tesis pregrado Maestría. Universidad Veracruzana, Xalapa, Veracruz]. http://cdigital.uv.mx/handle/123456789/40650

UNAM (2009). México frente a la crisis: hacia un nuevo curso de desarrollo. http://www.scielo.org.mx/scielo.php?script=sci arttext\&pid=S1665-952X2009000300001

Velazquez, K. (2016). Evoluciona la AMIPCI a la Asociación de Internet. https://marketing4ecommerce.mx/evoluciona-la-amipci-la-asociacion-de-internet/

Westreicher, G. (2017). Innovación empresarial. Economipedia haciendo fácil la economía: https://economipedia.com/definiciones/innovacion-empresarial.html

Xalapa, D. d. (2019). Finanzas / Veracruz, rezagado en acceso a Internet. https://www.diariodexalapa.com.mx/finanzas/veracruz-rezagada-en-acceso-a-internet$\underline{3268631 . h t m l}$

Xalapa, D. d. (2020). Arrasa el coronavirus a comercios xalapeños; no reabriría el 30\%. https://www.diariodexalapa.com.mx/local/arrasa-el-coronavirus-a-comercios-xalapenos-noreabriria-el-30-canaco-servytur-economia-pandemia-5318387.html 